\title{
The Digital Correlator Based on FPGA in PCS
}

\author{
Haiying Jiang , Yuliang Ma and Lu Zhang \\ School of Information Science and Engineering, University of Jinan, Jinan, China \\ ise_jianghy@ujn.edu.cn
}

\begin{abstract}
Based on the calculation theory of photon correlation spectroscopy and the internal structure on the digital correlator, the digital correlator is designed for the photon correlation spectroscopy. Using FPGA powerful computing function and abundant memory resources to achieve the 256 channel digital correlator, effectively improve the real-time and accuracy. At the same time, FPGA also enhances the integration and flexibility of the system.

Index Terms - Digital Correlator, FPGA, PCS.
\end{abstract}

\section{Introduction}

Photon Correlation Spectroscopy PCS is an effective method to measure the size of sub-micron particle. It is based on the relevance of the sequence of photon signal to detect the Doppler frequency shifter of the measured signal. As the Brownian motion of particles, the scattered light intensity is related to the average fluctuates stochastically in some fixed space positions, the speed of fluctuation is associated with the size of particles, the smaller particles, the faster fluctuation. PCS utilized the speed of fluctuation which the Brownian motion of particle to obtain the information of particle size ${ }^{[1,2]}$.

The response speed of digital correlator is a crucial technology in Photon Correlation Spectroscopy PCS, which directly affects the final result, requiring high-speed and highprecision digital correlator. The realization of digital correlator may use hardware and software. Generally, it uses the hardware to implement in abroad, but the price is expensive. The software approach of domestic is made data files with scattered light signal which gathered high-speed, and extracts the correlation function by computer, it is an "afterwards" method, does not have real-time. The hardware realization method of domestic first is photon digital correlator which composed by single-chip microcomputer and computer. Its development cost is low, but precision and real-time is poor, cannot meet the requirements for digital correlator that measure the sub-micron particle sizing currently ${ }^{[3]}$. After using DSP micro-controller which has real-time and high-speed, but compared with FPGA, low scalability and re-programming ability $^{[4-6]}$. At present, domestic digital correlator which was usually realized by FPGA has 64 and 128 channels. The more number of channels and the shorter sampling time, the higher experimental precision is ${ }^{[7]}$. In this paper, the design use the rich resources of FPGA to design the auto-correlation algorithm and to implement 256 channels, high-precision and flexible system to meet on the high-precision, real-time requirements in photon correlation spectroscopy.

\section{II . The Theory of Photon Correlation Spectroscopy}

The measurement principle diagram is shown in Fig. 1.The light emitted from the laser is as the light source, which focused by a lens issue strikes the particle samples of sample pool that is doing random thermal motion. When the laser irradiates the sample pool, scattering light comes out because of the interaction between particles and the electromagnetic filed of light wave ${ }^{[8]}$. At a fixed scattering angle, the scattered light of particles focused by a lens into the photomultiplier tube (PMT) which outputs photons signal after amplification and discriminating, transform it into the the same amplitude TTL serial pulse, and send to the subsequent digital correlator for correlation computation obtains intensity autocorrelation function. According to the particle information, the autocorrelation function contained the calculation results of the particle size distribution and show its nature ${ }^{[9,10]}$.

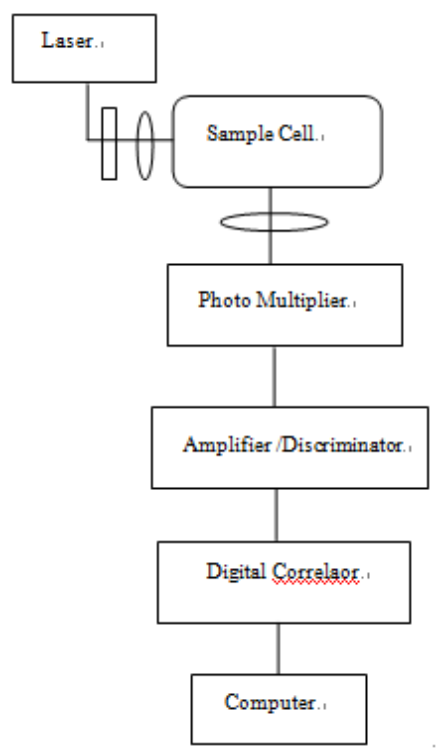

Fig. 1 PCS Particles Measurement System Diagram

When measuring the particles in the solution, if the same size and shape, the autocorrelation function of the scattering intensity is a simple exponential decay function, attenuation constants $\Gamma$ are inversely proportional to size:

$$
\Gamma=D q^{2}
$$

Where $D$ is the translational diffusion coefficient of particles, $q$ for the light scattering vector, can be expressed as:

$$
q=\frac{4 \pi n}{\lambda} \sin \left(\frac{a}{2}\right)
$$

For spherical particles, expressed by the Stokes-Einstein equation as follows: 


$$
D=\frac{K_{B} T}{6 \pi r \eta}
$$

According to the autocorrelation function of light intensity and more variety, as the particle size can be obtained:

$$
r=\frac{K_{B} T q^{2}}{6 \pi \eta \Gamma}
$$

\section{The Overall Design of Digital Correlator}

\section{A. Correlation Algorithm}

The digital correlation algorithm is based on the basis of discrete domain, the autocorrelation function of random signal in the digital system that is sampled and quantified on the expression is derived from the correlation function:

$$
\hat{R}_{x x}(\tau)=\frac{1}{\mathrm{~N}} \sum_{k=0}^{\mathrm{N}-1} x(k \Delta t) x(k \Delta t-\tau)
$$

Among them, $\mathrm{N}$ is the signal sample, $\Delta t$ is the sampling interval.

\section{B . FPGA Realization of Digital Correlator}

In this paper, in order to meet the requirements of high realtime and high precision, with the FPGA realized 256-channels digital correlator. The basic structure of digital correlator is shown in fig. 2 .

1) Input: the input signal is a serial pulse signal that generated from the photomultiplier tube.

2) Sampling Time Generator: Selecting an appropriate sampling time, a register width which is used to count the time interval, divide the input signal is into equal time interval, at each arrival time, the pulse counter pulse_count send the value to the shift register count_buffer, which put the value of pulse counter in real-time out.

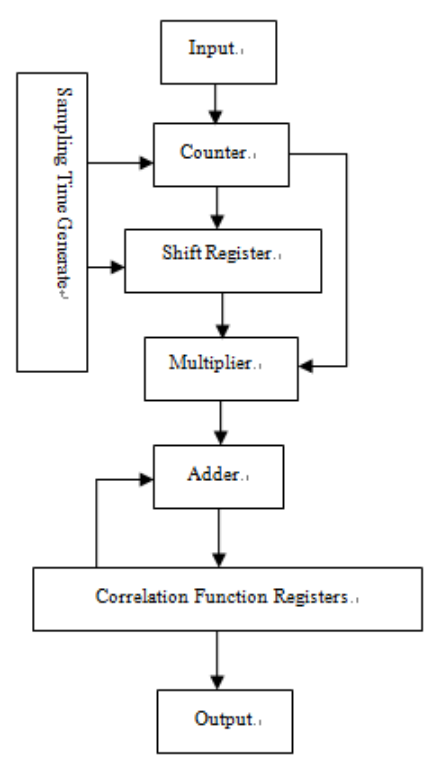

Fig.2 The Basic Structure of Digital Correlator
3) Shift Register Counter: Count_buffer pulse of each cycle the number of counts. At the end of each sampling time, the number in the shift register counter is sent into the first segment of the shift register, of which the data is moved to the second and so on ${ }^{[11]}$.

4) Multiplier and Adder: multiply the number of pulses from the counter by the number that is in the shift register for each section then accumulates the value of each channel.

5) Correlation Function Register: the correlation function register is divided into multiple storage channels, each discrete point value is stored in each channel. At the same time, the number of the pulses obtained from the counter is sent to the multiplier and the shift register, add the value after multiplying, then send the final results into the correlation function re-register ${ }^{[12]}$. The accumulated value of $m$ channel registers is:

$$
C(m \Delta t)=\sum_{j=1}^{N} n_{j} n_{j+m}
$$

The $m$ is the channels number of digital correlator, $n_{i}$ is the pulse number of the $i$ segment in shift register, the first channel of the accumulated value-added namely $n_{1} n_{2}+n_{2} n_{3}+n_{3} n_{4}+\cdots$.

Using the powerful arithmetic functions and rich digital storage resources of FPGA to realize digital correlator, this advantage could greatly simplified in a chip with some original complex operation and digital logic ${ }^{[13]}$.

This design uses the Verilog language program, the algorithm is as follows:

begin: $\mathrm{m} 2$

for $(\mathrm{i}=\mathrm{M}, \mathrm{i}>0, \mathrm{i}=\mathrm{i}-1 \mathrm{\prime} \mathrm{b} 1)$

begin

count_buffer[i]=count_buffer[i-1'b1]

cor_buf $[\mathrm{i}]=$ cor_buf[i-1'b1]

end

end

count_buffer[0]=pulse_count

cor_buf[0]=pulse_count

pulse_count $<=6$ 'b00_0000,//cleared, the next count of clock interval

$\mathrm{g} 2<=\mathrm{g} 2+1$ 'b1, //control the number of Multiplier

if $(\mathrm{g} 2<\mathrm{N})$

begin: $\mathrm{m} 3$

for $(\mathrm{g} 1=1, \mathrm{~g} 1<=\mathrm{N}, \mathrm{g} 1=\mathrm{g} 1+1$ 'b1)

chengfaqi $[\mathrm{g} 1]=$ chengfaqi $[\mathrm{g} 1]+$ cor_buf $[0] *$ cor_buf $[\mathrm{g} 1]$

end

else if $(\mathrm{g} 2<\mathrm{g} 4)$

begin

begin: $\mathrm{m} 4$

g5 $=\mathrm{g} 2-\mathrm{N}$

for $(\mathrm{g} 1=1, \mathrm{~g} 1<=\mathrm{N}, \mathrm{g} 1=\mathrm{g} 1+1$ 'b1)

begin 
chengfaqi $[\mathrm{g} 1+\mathrm{g} 5]=$ chengfaqi $[\mathrm{g} 1+\mathrm{g} 5]+$ cor_buf $[0] *$ cor_bu $\mathrm{f}[\mathrm{g} 1+\mathrm{g} 5]$

end

out=chengfaqi $[\mathrm{g} 5+1$ 'b1]

number $=\mathrm{g} 5+1 \mathrm{\prime} \mathrm{b} 1$

out_flag $<=1$ 'b1

end

end

Simulation waveforms are shown in Fig.3, Fig.4 and Fig.5:

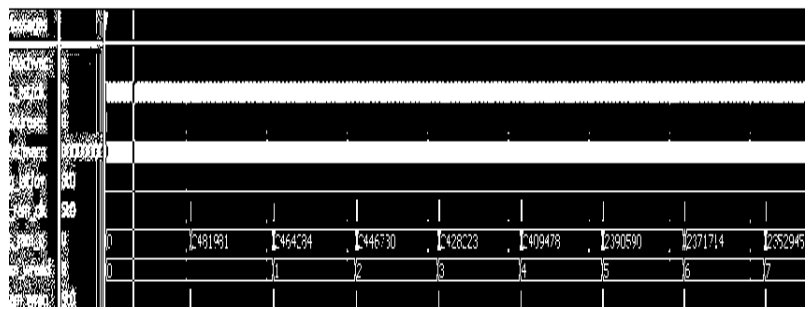

Fig. 3 Simulation Waveform-1

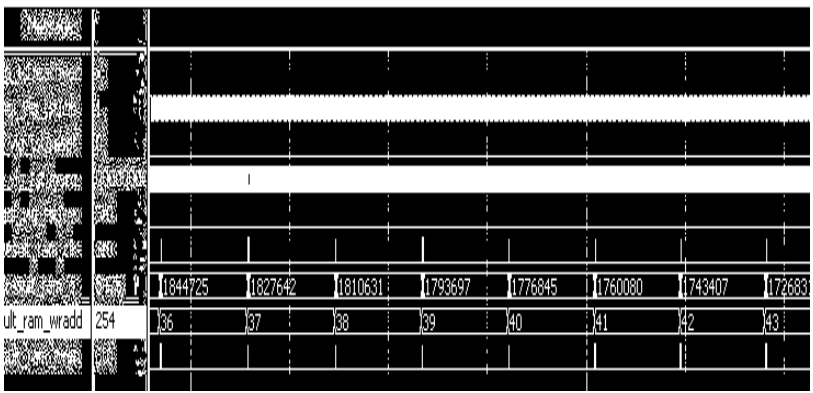

Fig. 4 Simulation waveform-2

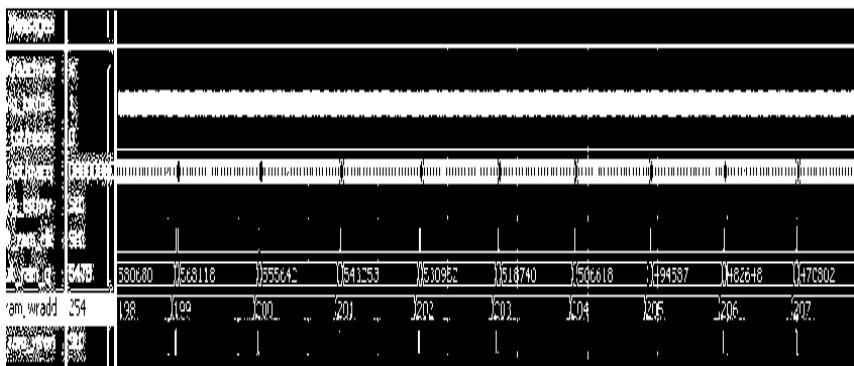

Fig. 5 Simulation waveform-3

256 channels with Matlab verify the accuracy of digital correlator, the following was relatively Fig.3-Fig.5, simulation results of the test results:

$$
\mathrm{y} 256=\mathrm{x} \operatorname{corr}(\mathrm{x} 256)
$$

$\begin{array}{rrrrrr}2481981 & 2464284 & 2446730 & 2428223 & 2409478 & 390590 \\ 1844725 & 1827642 & 1810631 & 1793697 & 1776845 & 1760080 \\ 1072983 & 1067503 & 1062236 & 1057181 & 1052337 & 1047703\end{array}$

\section{IV . Conclusion}

PCS, a photon spectroscopic technology, is an effective way for the measurement of sub-micron and micron particle size. Compared to other countries, relative researches of our nation were late. This technology requires high-channel-count digital correlator, and in this paper, 256 channels were achieved with the FPGA technology. In another aspect, the realization of 256 channels also greatly improved the cogency of the relevant experimental results. Delay through width register and easily adjustable by re-programmed features of FPGA. At the same time, FPGA is also one of the best choices to improve the integration and reliability of systems. It is an integrated circuit chip, with features of large-scale, low-power, re-programmed and supporting in-system programming. A great deal of flexibility nature was provided for the system development, and the variety of digital signal processing, stability and real-time were achieved.

\section{References}

[1] Shen Jin, Zheng Gang, Li Mengchao and Sun Guoqiang. "The Study on the Estimation of Autocorrelation Function in PCS Particle Sizing Technique," Chinese Journal of Scientific Instrument, Vol. 24, No. 6, 2003, pp. 585-588.

[2] Theodore Provedr. "Chalenges in particle size distribution measurement past, present and for the 21st century," Progress in Organic Coalings, No. 32, 1997, pp. 143-153.

[3] Zhou Peiling, Peng Hu and Yao Kun. "SCM in the photon correlator application," Modern Electronic Technique, No. 4, 1994, pp. 26-28.

[4] Li Haisen. "A parallel algotithm of correlation processing and its hardware architecture realization," Acta Acustica, Vol. 5, No. 19, 1994, pp. 361-366.

[5] Hu Hui and Ding Shiqi. "Parallel Achevement of Digital Correlation and Convolution Based on TMS320C80," Journal of Data Acquisition Processing, Vol. 14, No. 4, 1999, pp. 457-461.

[6] Zhang Mintao. "Implement of Digital Correlator Using DSP+FPGA." Modern Electronic Technique, No. 9, 2006, pp. 72-74.

[7] Wang Yuan. "Research about Sub-micro Particles Sizing Based on Dynamic Light Scattering [D]. Chongqing: Chongqing University, 2005.

[8] Guo Yongcai, Wang Yuan, Gao Chao and Deng Dongmei. "Particle Sizing with Dynamic Light Scattering." Journal of Chongqing University, Vol. 29, No. 2, 2006, pp. 35-37.

[9] Chen Weiping, Dong Xueren, Xu Tao and Wang Shaoqing. "Research on PCS Nano-particle Size Measuring Apparatus," Instrument Technique and Sensor, No. 10, 2006, pp. 24-26.

[10] Shen Jin, Zhen Gang, Sun Hao and Yang Yuchuan. "PCS Particle Sizing Instrument," Instrument Technique, No. 2, 2003, pp. 7-8.

[11] Lei Tangcai, Lin Ke and Huang Yanlei. "Study on Correlation in Photon Correlation Spectroscopy," Instrument Technique, No. 6, 2006, pp. 2526.

[12] Lin Ke and Huang Yanlei. "Study on the Correlation in Photon Correlation Spectroscopy," Chinese Journal of Scientific Instrument, Vol. 27, No. 6, 2006, pp. 174-176.

[13] Dong Liang, Wang Min, Chen Jianhua and Miao Aimin. "Design of Embedded Multi-bit Autocorrelator Based on FPGA," Modern Electronic Technique, No. 18, 2009, pp.20-24 